\title{
DAMAGE DETECTION IN COMPOSITE BEAM USING NUMERICAL MODAL ANALYSIS
}

\author{
Ramanamurthy E.V.V. ${ }^{1}$, Chandrasekaran K. ${ }^{2}$ \\ ${ }^{1}$ Research Scholar, Department of Mechanical Engineering, JNTU, Hyderabad, India \\ ${ }^{2}$ Department of Mechanical Engineering, R.M.K. Engineering College, Chennai, India \\ E-mail : evvrm.mech@gmail.com
}

\begin{abstract}
There is a pressing need to develop effective techniques for structural health monitoring (SHM), so that the safety and integrity of the structures can be improved. The main objective of this study is to evaluate the damage detection techniques for the composite beam. Cost-effective and reliable damage detection is critical for the utilization of composite materials. The study is carried out both analytical and experimental methods for the detection of damage in composite materials and the results are presented in this paper. A crack in a structure will affect the modal parameters both locally as well as globally. The present work discusses the studies carried out using those parameters that get changed locally and globally as well as a procedure to identify the crack location. Structural damage detection has gained increasing attention from the scientific community since unpredicted major hazards, most with human losses, have been reported. Aircraft crashes and the catastrophic bridge failures are some examples. Since damage alters the dynamic characteristics of a structure, namely its eigen properties (natural frequencies, modal damping and modes of vibration), several techniques based on experimental modal analysis have been developed in recent years. A cantilever composite beam was considered and modeled. The crack was introduced and analyzes the propagation of the crack. The crack is a through-thickness one, growing along the breadth of the beam starting from one edge. The location of the crack is also moved from the fixed end to the free end along its length. The changes in natural frequencies are observed from analytical study, due to the presence of the crack at different locations and depths and 2Dfinite element models were created the percentage change in frequency values are calculated. These results are confirmed by the experiments. The numerical finite element $(\mathrm{FE})$ analysis is performed to complement the damage detection by using different algorithms. Due to refined analysis of $\mathrm{FE}$ approach, all the algorithms are viable of detecting the damage using the FE data. The add-on advantage of the damage detection algorithms (Gapped Smoothing Method and Strain Energy Method) is that the data from the healthy beam are not required as the reference, thus simplifying the detection process.
\end{abstract}

Key words: Damage algorithm, Central Difference Approximation, Curvature mode Shapes, damage Index, Curvature damage factor, Strain energy method, Gapped Smoothed Method

\section{INTRODUCTION}

Damage can cause structural failure, and sudden failure during high load operation may lead to catastrophic consequences. Development of an early damage detection method for structural failure is one of the most important keys in maintaining the integrity and safety of structures. The cracks can be present in structures due to their limited fatigue strengths or due to the manufacturing processes. These cracks open for a part of the cycle and close when the vibration reverses its direction. These cracks will grow over time, as the load reversals continue, and may reach a point where they pose a threat to the integrity of the structure. As a result, all such structures must be carefully maintained and more generally, SHM denotes a reliable system with the ability to detect and interpret adverse "change" in a structure due to damage or normal operation.

The greatest challenge in designing a SHM system is to identify the underline changes due to damage or defect. Lots of damage detection techniques have been proposed for structural health monitoring. Some of the nondestructive evaluation approaches that utilize technologies such as X-ray imaging, ultrasonic scans, infrared thermograph, and eddy current can identify damages. However, they are somehow difficult to implement, and some of them are impractical in many cases such as in service aircraft testing and in-site space structures. Almost all of the above techniques require that the vicinity of the damage is known in advance and the portion of the structure being inspected is readily accessible. The drawbacks of current inspection techniques have led engineers to investigate new methods for continuous monitoring and global condition assessment of structures. That is the case for methods based on vibration responses that allow one to obtain meaningful time and/or frequency domain data and calculate changes in the structural and modal properties, such as resonance frequencies, modal damping and mode shapes, and use them with the objective of developing reliable techniques to detect, locate and quantify damage. 
The dynamic response of structures can offer unique information on defects that may be contained within the structures. Changes in the physical properties of the structures due to damage will alter the dynamic responses such as natural frequencies, damping and mode shapes. These physical parameter changes can be extracted to estimate damage information. In the past 20 years a lot of work has been published in the area of damage detection, where various methods have been proposed. The modal parameters such as natural frequencies and mode shapes can be used to detect the initiation and development of cracks. In this present work, different damage algorithms were used to locate the crack in a cantilever composite beam.

\section{LITERATURE REVIEW}

The dynamics-based damage detection is an effective method of acquiring both the global and the local information of the structure. Significant efforts have already been spent to develop damage detection algorithms using dynamics-based approach. Some of the researches related to damage detection using dynamicsbased approach are summarized as follows.

\section{A. Frequency-based Damage Detection}

Natural frequency-based method may be the easiest one of dynamics-based damage detection due to ease of measurement of the natural frequencies. In many applications only a single sensor and a single point of measurement are required. The foundation of this method is that damage produces a change in structural stiffness, which in turn, results in changes of natural frequencies compared to the healthy or intact structures. Therefore, the measurement of frequencies at different modes could be used to probe possible damage locations. The frequency-based method for damage identification was successfully applied to simple laboratory structures with only single or a few damage locations. The frequencybased method is usually reliable for detecting the presence of damage in a simple composite structure, whereas the important information about damage size and type, location, and orientation cannot be obtained using this simple method since several combinations of these variables can yield similar or identical frequency change.

\section{B. Mode Shape-based Damage Detection}

A more robust application of dynamics-based approach for damage detecting is based on mode shapes. This method has been developed to assess damage directly using the measured displacement mode shapes or curvatures mode shapes. The displacement mode shapes were determined experimentally using accelerometers and analytically using E-B beam theory, and their comparison showed that the crack location could be found and the crack depth was estimated with satisfactory accuracy.

A potential disadvantage of mode shape-based method is that it needs to measure

a large number of points. A more effective method of damage detection based on the mode shapes is the use of curvature mode shapes. From Euler-Bernoulli beam theory, the curvature mode shape $(\mathrm{K})$ is related to the Young's Modulus of beam and the beam cross sectional geometric properties.

The use of curvature mode shapes in damage identification is based on the assumptions that the curvature of an undamaged structure is smooth and continuous and the irregularity of the curvature can thus determine the location of the damaged for a homogeneous structure. The changes in the curvature mode shapes are highly localized to the region of damages, and they are more pronounced than the changes in the displacement mode shapes. The curvature is often calculated from the measured displacement mode shapes by using a central difference approximation.

The earlier studies on dynamic response based damage detection used the natural frequency as a parameter by many researchers - ( Adam et al., 1978; Cawley and Adams,1979a, b; Gomez and Silva, 1991; Salawu, 1997; Messina, A., Williams, E.J. and Contursi, T, 1998; Zhou et al. 2000) reviewed some of the vibrationbased detection techniques for identification of delamination in composite structures. Lee and Chung (2000) used the first four frequencies of a simulated cantilever beam to locate a single crack and assess their corresponding effectiveness. The crack depth was then approximated iteratively to match the frequency as closely as possible before the location of the crack was finally defined. Chinchalkar (2001) developed a numerical method for determining the crack location in a damaged beam. A rotation spring was used to model the beam. The graphs of spring stiffness versus crack location were plotted for three natural frequencies, and the point of intersection of the three curves indicated the location of the crack. Lestari and Hanagud (2001) used only a few lower order curvature modes to identify damage location based on the difference between the measured data of damaged and healthy structures, and the mathematical relationship of the measured data and dynamic parameters was used 
to estimate the severity of the damage. The effect of damage on frequency response primarily provides global information about the condition of structures. Using a small number of sensors, the natural frequencies of large structures can be measured effortlessly. The natural frequency measurements are very sensitive to interference, especially at the low order modes. Some of the examples of interferences include fiber misalignment during manufacturing, introduction of non-negligible mass by sensors, and simulated approximate boundary conditions that prompt the largest error in the frequency measurement (Kessleret al., 2002).Recently, Lestari and Qiao (2005) employed the curvature-based method to detect the damage in honeycomb composite sandwich beams. The FRF curvature methods performed well in detecting and locating the damage, especially for large magnitude of damage.

The natural frequency can be used as an early warning system of global structural condition. The mode shapes are utilized as a base parameter for damage identification and assessment technique in the present study. The changes of the curvature mode shapes are localized in the region of the damage and consequently may be used effectively to identify damage location in structures.

This detection technique is extended in the present study, and the amplitude difference of curvature mode shapes is employed to estimate the local stiffness loss due to damage. The first curvature mode is used in the damage assessment, since the curvature nodal points of damaged structures at high modes may shift significantly from the original undamaged case thus generating misleading results. It is relatively difficult to experimentally obtain the mode shapes at the higher modes using surface-bonded sensors; while at the lower modes, the mode shapes may not be sensitive to small damage. In this study, the first three low curvature mode shapes of the structures between an undamaged state and a damaged state are extracted from the finite element analysis data. Then, the differences of these curvature modes are used to determine damage locations and the corresponding magnitudes. The procedure illustrates the damage state from the original undamaged state; however, it can also be modified to consider the changes from one damaged state to another damaged state. The study is conducted by using Glass / Epoxy laminated composite beam, and the curvature mode shapes are measured by using FEM software ANSYS. Several damage algorithms forms are introduced and the results are plotted. The results of damage identification for the Glass / Epoxy laminated beam are presented.

\section{EVALUATION OF DAMAGE DETECTION}

In present work both the experimental as well as FEM numerical procedures are used to determine the frequency changes before they are compared for different algorithms. A study is made to compare the change in frequencies, obtained due to the presence of a crack in a composite beam with an uncracked beam. The general purpose FEM analysis software ANSYS was used in the modal analysis module to obtain mode shapes. Due to the increasing confidence in the procedure, the finite element analysis was extended to more crack locations and crack ratios to obtain the change in frequencies as the crack moves from fixed end to free end in closely spaced intervals. In the analysis, the crack location was assumed to move from $0.1 \mathrm{~L}$ to $0.8 \mathrm{~L}$ and the crack depth assumed to move from $0.1 B$ to $0.5 B$, where $L$ and $B$ represents the length and breadth of the composite beam.

The following Damage detection algorithms were used to locate the damage in the composite beam buy using FE analysis. The modal parameters that change locally are mode shapes, i.e., bending mode shape and torsional mode shape.

1. The Curvature Mode Shape Method,

2. The Damage Index Method,

3. The Curvature Damage Factor,

4. The Gapped Smoothing Method.

5. The Strain Energy Method,

The above methods are used in the evaluation of damage in the composite beam for bending and torsion mode shapes. The results obtained were tabulated, plotted and discussed in the next section

\section{The Curvature Mode Shape :}

Pandey, et al. (13) Stated that, the mode shapes of a damaged and the corresponding undamaged structure are identified, the curvature at each location $i$ on the structure is numerically obtained by central difference approximation.

$$
\phi^{\prime \prime} i j=\left(\phi_{(i+1), j}-2 \phi_{i j}+\phi_{(i-1), j}\right) / h^{2}
$$


Where, $i$ is the node number, $j$ is the mode shape number and $\mathrm{h}$ is the distance between the measurement points $i+1$ and $i-1$.

The location of the damage is then identified by the largest computed absolute difference between the mode shape curvatures of the damaged and undamaged structure, as follows

$$
\left.\left\{\Delta \phi_{i j}{ }^{\prime}\right\}=\mid\left\{\phi_{i j, d}\right\}^{\prime}\right\}-\left\{\phi_{i j, u d}\right\} \mid
$$

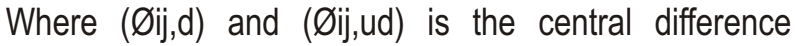
approximation of damaged and undamaged structure respectively

\section{The Damage Index Method:}

Stubbs and Kim (14) stated that, the damage identification based on the changes in the curvature of the $j$ th mode at location $i$. The damage parameter formulation of this method is presented as

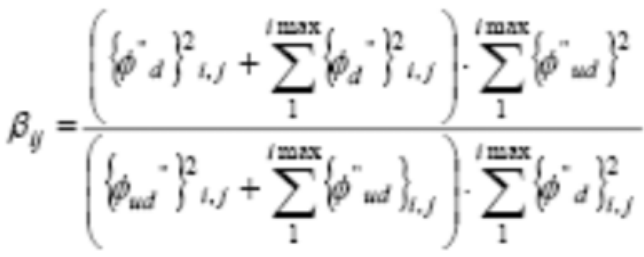

where $\beta_{i j}$ is the Damaged Index at location $i$ for mode $j$.

\section{The Curvature Damage Factor:}

Wahab and De Roeck (15), successfully applied a curvature - based method to the Z 24 bridge in Switzerland and introduced a damage indicator named the curvature damage factor. The difference in curvature before and after damage averaged over a number of considered modes is defined as the CDF

$$
C D F=\frac{1}{N} \sum_{i}^{l}\left|\dot{\phi_{w d i}}-\dot{\phi_{d, i}}\right|
$$

\section{The Gapped Smoothing Method:}

Ratcliff and Bagaria (16) proposed the gapped smoothing method for the damage detection in a beam without prior data of the undamaged structure. The basic theory of this method is that the mode shape of the undamaged structure has a smooth curve and it can be approximated by a polynomial in one variable.

$$
U(x)=\sum_{i=0}^{n} a_{i} x^{n-i}
$$

The damage parameter based on this method is defined as the square of the difference between measured data of the damaged structure and the smoothed fitted value.

The maximum value of this method indicates the damage location. This method does not require an undamaged structure reference. The procedure mainly depends on the measured mode shape obtained from damaged structure. The main advantage of this method is, that it can be applied to an existing structure where there is no prior data of its undamaged structure.

The displacement mode shape of a damaged structure is to be converted to curvature shape by using central difference approximation. Then using a gaped polynomial at each point to generate mode shapes, which representing the healthy beam, locally smoothes the curvature mode. The Damage index by this method was defined as the difference between the curvature and the gapped smooth polynomial at each point. The largest index represents the location of the damage.

$$
\text { DamageIndex }=\mathrm{D}_{\mathrm{GSM}}(x)=\left(U_{\text {meanwed }}(x)-U_{\text {fitung }}(x)\right)^{2}
$$

\section{The Strain Energy Method:}

A certain amount of strain energy is stored in a particular vibration mode, the frequency and mode shapes are highly sensitive to changes in the stiffness of the structure, which are associated with the strain energy. Hence the change in the modal strain energy may also be considered as an indicator for the damage location. The damage indicator for this method is defined as the absolute difference between the square of measured damage data and square of the smoothed fitted curvature value. 
The strain energy $\left(U_{i}\right)$ associated with a particular mode shape at one segment of the beam and may be calculated as

$$
U_{i}=\frac{1}{2} \int_{\mathrm{x}_{\mathrm{k}}}^{\mathrm{x}_{\mathrm{k}-1}} E I\left(\frac{\partial^{2} \phi_{l}}{\partial x^{2}}\right) d x=\frac{1}{2} \int_{\mathrm{x}_{\mathrm{k}}}^{\mathrm{x}_{\mathrm{k}-1}} E I k_{i}^{2} d x
$$

where $\emptyset_{\mathrm{i}}$ is the displace me nt mode shape;

$k$ is the curvature mode shape;

El is the bending stiffness of the structure

The curvature in strain energy and the beam bending stiffness are interrelated, the damage-induced stiffness reduction leads to increasing of curvatures. Therefore, the above equation (7) my also be considered as a logical choice of indicator for the damage location. For this calculation the curvature required is extracted from the displacement mode shapes measured using FEM ANSYS by using a central difference approximation.

The damage Index using the concept of strain energy is proportional to the square of the curvature. The damage index can be calculated by using the eq(8). This method is defined as the absolute difference between the square of measured data and square of the smoothed fitted curvature Values eq (8).

$$
\text { Damage Index }=\left|k_{\text {mearured }}(x)^{2}-k_{\text {fintugg }}(x)^{2}\right|
$$

\section{EXPERIMENTAL PROCEDURE}

In the present work, experimental and numerical (FEM) procedures are used to evaluate the variation of frequency change before they are compared. The specimen used in this study was made of fiber glass and epoxy resin using hand lay-up process. It has the dimensions of length $450 \mathrm{~mm}$, width $30 \mathrm{~mm}$ and thickness $10 \mathrm{~mm}$. The laminated composite has a 45 fiber orientation lay-up for a total of ten layers. When the beam is clamped as cantilever configuration, the specimen has a free length of $400 \mathrm{~mm}$. The specimen dimensions for the experiment with a ratio $L: B: T$ as 40:3:1 as shown in the Fig. 1.

RION SA-73 Sound and Vibration Dual Channel analyzer is used to extract the natural frequencies, along with B\&K - Type 4344 accelerometer pickup. An Impact hammer RION PH-51 is used to excite the beam. Including fundamental frequency and few lower order frequencies were extracted from the experiment.

$L=$ Length of Beam $=400 \mathrm{~mm}$;

$B=$ Width of Beam $=30 \mathrm{~mm}$;

$\mathrm{T}=$ thickness of Beam $=10 \mathrm{~mm}$;
$L_{1}=$ Crack location from fixed end $=40 \mathrm{~mm}$;

$\mathrm{a}=$ Depth of Crack $=1 \mathrm{~mm}$ to $15 \mathrm{~mm}$

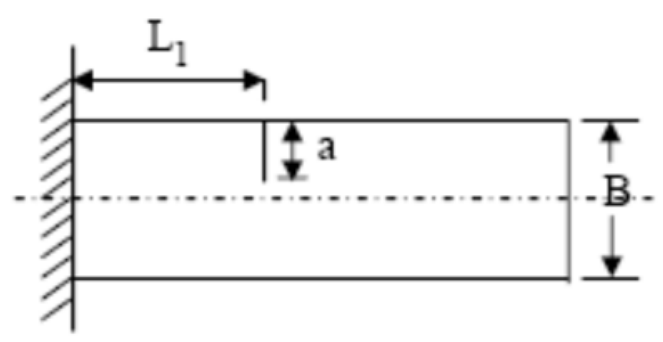

Fig. 1. Geometry of the cantilever composite beam with an edge crack.

Initially, the natural frequencies of uncracked composite cantilever beam were extracted. Without disturbing its setting, a crack was generated and propagated by means of a thin saw cut (around $0.8 \mathrm{~mm}$ thick). The experiment was conducted on Four specimens with the same geometry, material and creating a crack at $0.2 \mathrm{~L}$ to to $0.8 \mathrm{~L}$ with an increment of $0.2 \mathrm{~L}$ from the fixed end by varying crack depths of 0.1 to 0.5 , with an increment of $0.1 \mathrm{~B}$, at each location.

From the experiment, the measured values of frequencies are used to calculate the percentage change in the first three bending modes with respect to the uncracked beam for each location and depth These results are then compared with the numerical finite element results and discussed in the next section.

\section{NUMERICAL STUDIES USING ANSYS}

A Cantilever composite beam with the same geometry and material properties was considered for the numerical studies by using ANSYS software. The geometrical characteristics, the length $L$ ), Width (B) and thickness(T)of composite beam were chosen as $400 \mathrm{~mm}$, $30 \mathrm{~mm}$ and $10 \mathrm{~mm}$ respectively. The material properties of the glass-epoxy composite beam in terms of fibers and matrix, identified by the indices fand $m$, respectively, are:

$\begin{array}{ll}\text { Modulus of elasticity } & \mathrm{E}_{\mathrm{m}}=3.45 \mathrm{GPa}, \\ & \mathrm{E}_{\mathrm{f}}=72.4 \mathrm{Gpa}, \\ \text { Modulus of rigidity } & \mathrm{G}_{\mathrm{m}}=1.277 \mathrm{Gpa}, \\ & \mathrm{G}_{\mathrm{f}}=29.67 \mathrm{Gpa},\end{array}$

Poisson's ratio $\quad v_{\mathrm{m}}=0.22 ; v_{\mathrm{f}}=0.35$,

Mass density $\rho_{\mathrm{f}}=2600 \mathrm{~kg} / \mathrm{m}^{3} ; \rho_{\mathrm{m}}=330 \mathrm{~kg} / \mathrm{m}^{3}$;

Initially, the location of the crack and depth of crack were considered similar to the experimental procedure. The percentage change in frequency with respect to crack 
Ramanamurthy et al : Damage Detection in Composite Beam Using...

locations and crack depths were calculated and represented in the Fig. 2 First three bending modes were extracted and compared with the experimental values. Since the results are encouraging the numerical procedure is extended to more crack locations and crack depths to verify the change in frequencies. From the numerical analysis, the mode shape displacements are calculated at these frequencies. By using mode shapes data the damage detection was carried out by using damage algorithms (explained in the previous section). The numerical analysis was considered only for bending mode shapes. By using numerical procedure it is possible to extend the same procedure for extracting torsional mode shapes also.

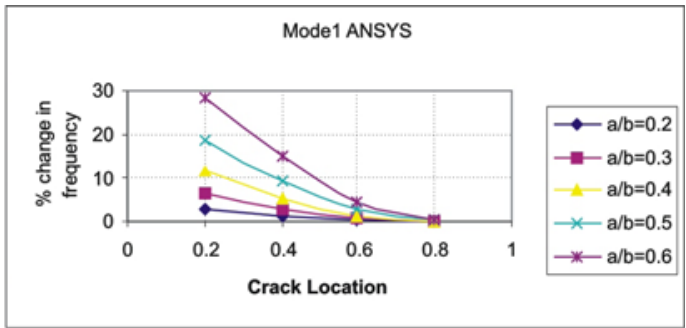

Fig. 2(a)

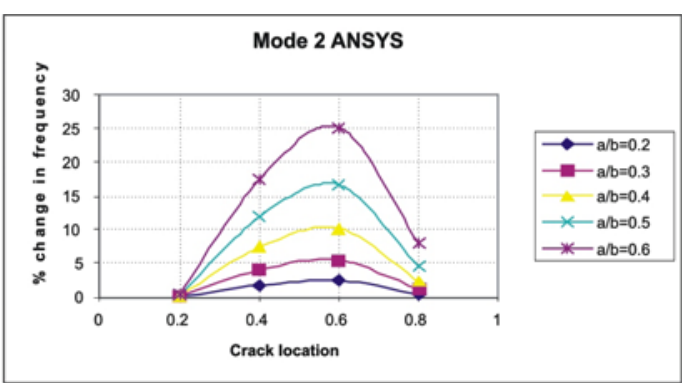

Fig. 2(b)

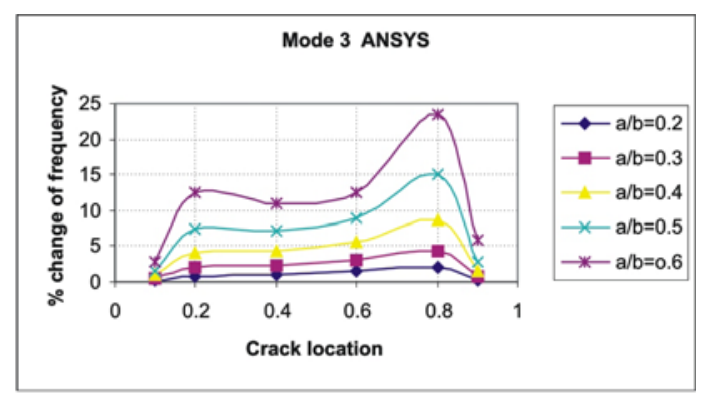

Fig. 2(c)

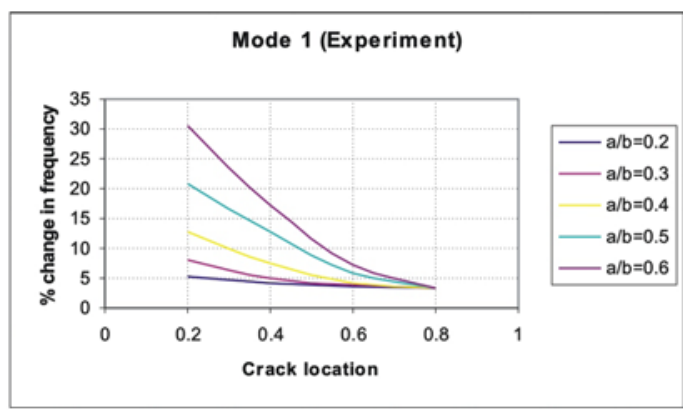

Fig. 2(d)

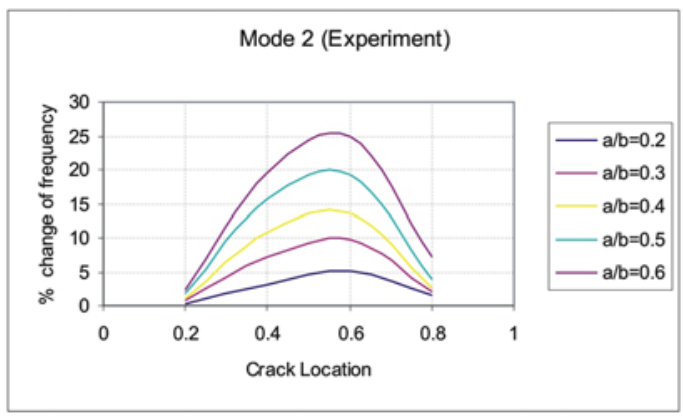

Fig. 2(e)

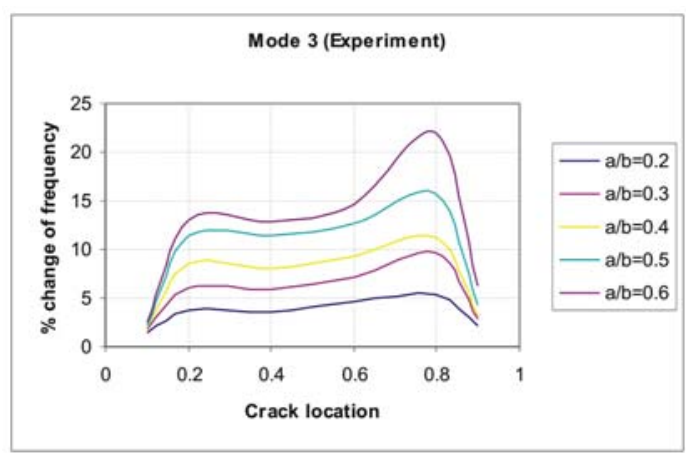

Fig. 2(f)

Fig. 2. Percentage of change in frequency in first three bending frequencies due to a crack ANSYS [2 (a),(b),(c)] and Experimental [2 (d),(e),(f)] results

Numerical modal analysis based on the finite element (FE) modeling is performed for studying the dynamic response of a structure. The natural frequencies and mode shapes are important modal parameters in designing a structure under dynamic loading conditions. The numerical analysis is carried out by using the commercial finite element program ANSYS. It is mainly used to verify the effectiveness of damage detection algorithms used in this study. 
In the present study, since the specimen is made of fiberglass/epoxy composite material, the FE modeling of the composite beam is simulated with the layered element (shell 99). The composite plate consists of several orthotropic layers, and it is considered as orthotropic material. The FE analysis software ANSYS was used in the modal analysis to obtain mode shapes.

A Cantilever beam of the dimensions L: B: T of composite material was considered for the numerical analysis. The beam was modeled with eight nodded shell elements so as to introduce the crack and analyze for the propagation of the crack.

\section{ANALYTICAL PROCEDURE RESULTS}

Numerical modal analysis based on the finite element (FE) modeling is performed for studying the dynamic response of a structure. In the present study, since the specimen is made of fiberglass/epoxy composite material, the FE modeling of the composite beam is simulated with the layered element (shell 99). The present study focuses on the identification of the damage in the composite beam by extracting the modal parameters obtained from the results of modal analysis by using the numerical finite element analysis and verify the validity of the damage detection algorithms.

Damage Index by using different damage algorithms:

The crack location from fixed end $L_{1}=40 \mathrm{~mm}$ and crack ratio $\mathrm{a} / \mathrm{b}=0.1$ ( i.e., depth of crack as $3 \mathrm{~mm}$ ) is considered.

For the comparison, the crack location from fixed end $=L_{1}=40 \mathrm{~mm}$ and crack ratio $\mathrm{a} / \mathrm{b}=0.1$ (i.e., depth of crack as $3 \mathrm{~mm}$ ) is considered. From the Fig. 3 , it is clear, that the Gapped smoothing method was higher performance than the Damage index method.

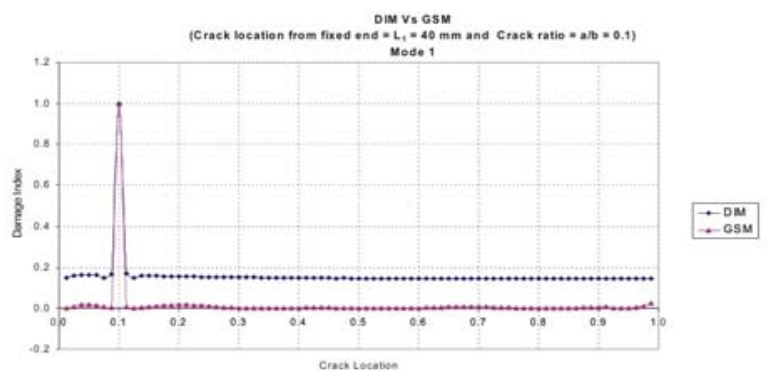

Fig. 3. Comparison between damage index method and gapped smoothing method.

For the comparison, the crack location from fixed end $=L_{1}=40 \mathrm{~mm}$ and crack ratio $\mathrm{a} / \mathrm{b}=0.1$ (i.e., depth of crack as $3 \mathrm{~mm}$ ) is considered. From the Fig. 4 , it is clear, that the Strain energy method was higher performance than the Damage index method.

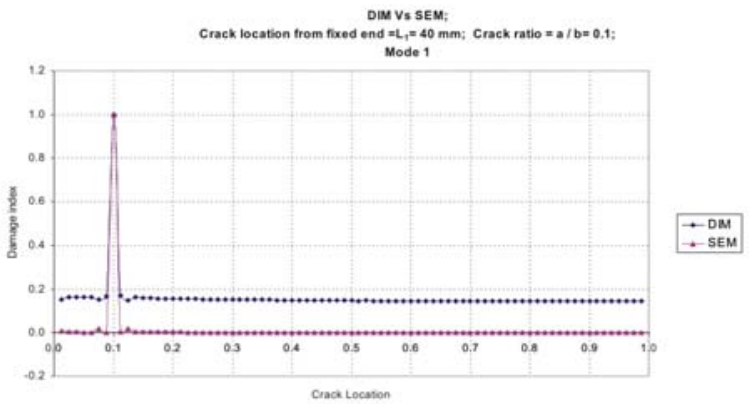

Fig. 4. Comparison between damage index method and strain energy method

\section{DISCUSSIONS}

The different damage detection algorithms ( Curvature mode shape method, Damage index method, Curvature damage factor method, Gapped smoothing method, Strain energy method) are employed to analyze the numerical data. The crack location and damage index values for the damage detection algorithms for the above methods are represented in the figures listed below

\section{CURVATURE MODE SHAPE :}

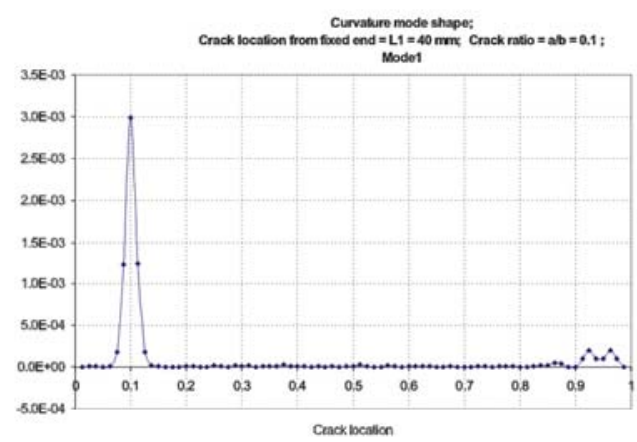

Fig. 5(a)

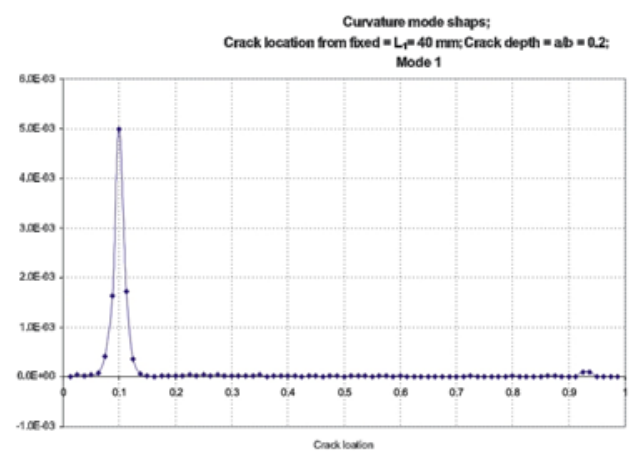

Fig. 5(b) 


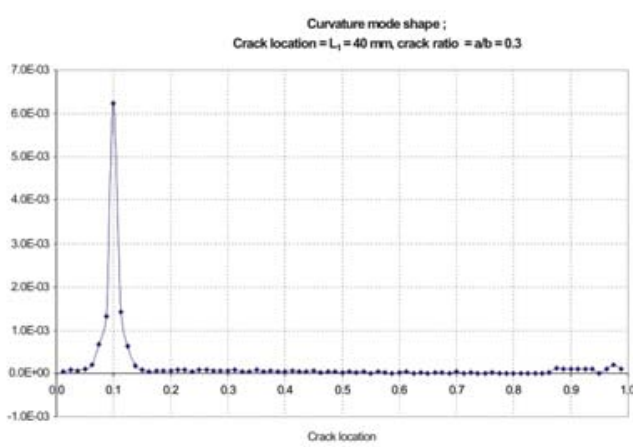

Fig. 5(c)

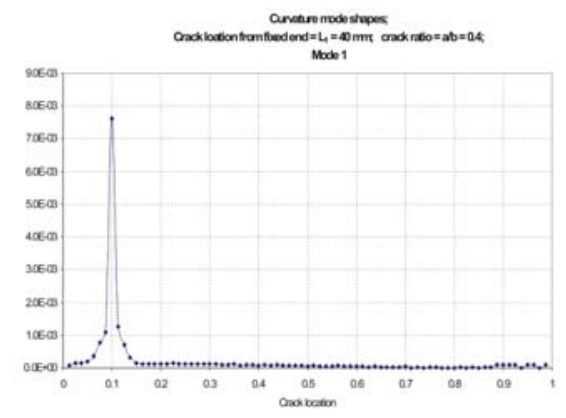

Fig. 5(d)

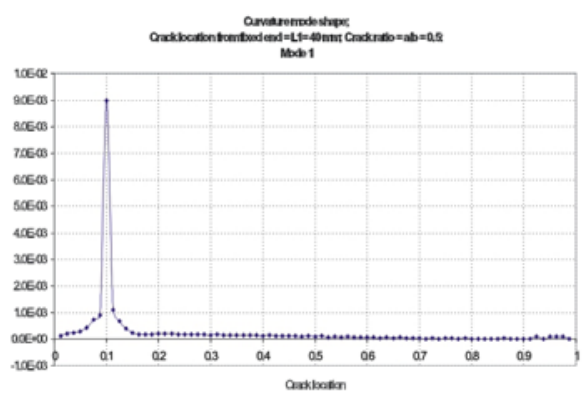

Fig. 5(e)

2. DAMAGE INDEX METHOD :

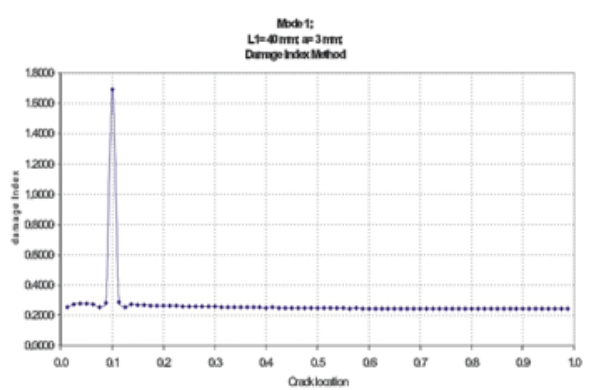

Fig. 6(a)

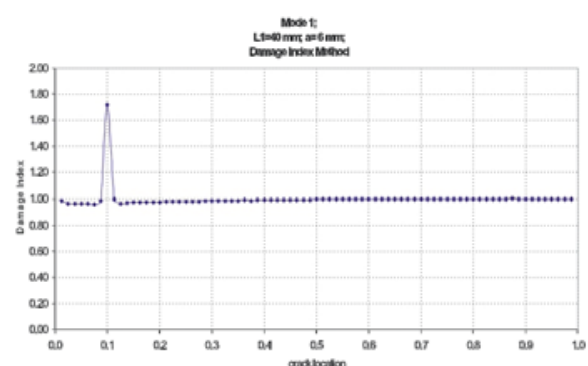

Fig. 6(b)

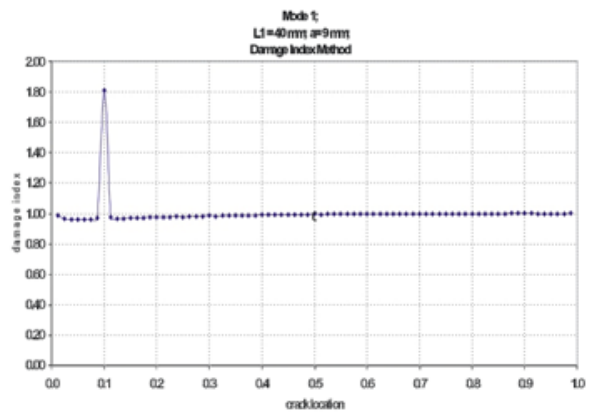

Fig. 6(c)

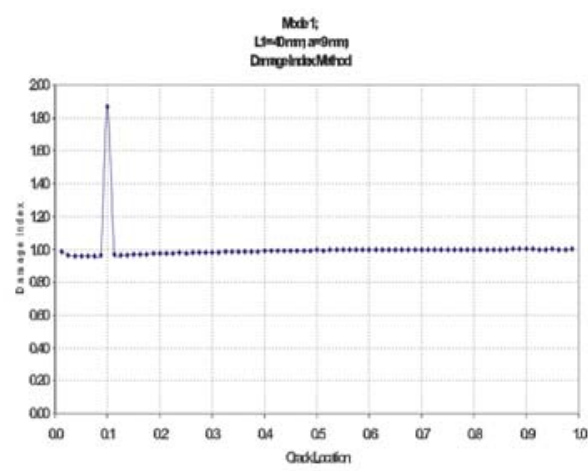

Fig. 6(d)

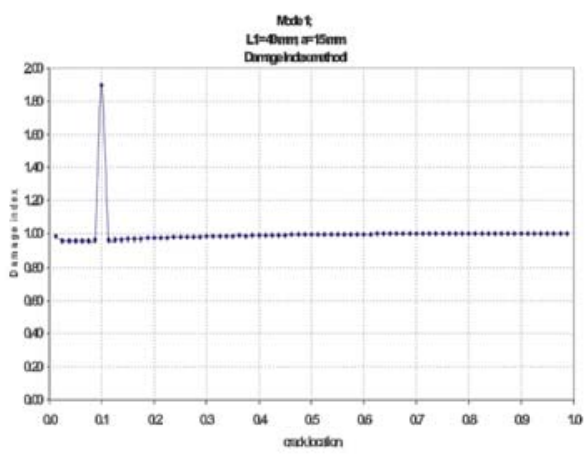

Fig. 6(e) 
3. CURVATURE DAMAGE FACTOR :

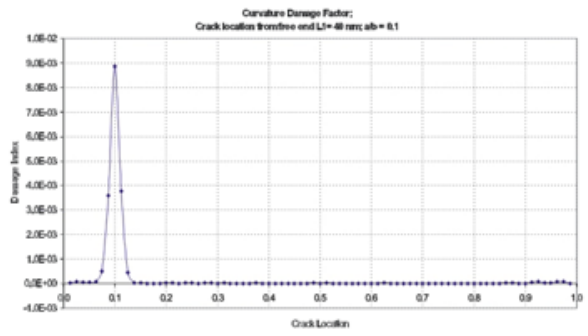

Fig. 7(a)

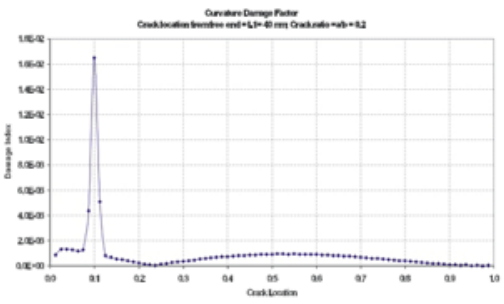

Fig. 7(b)

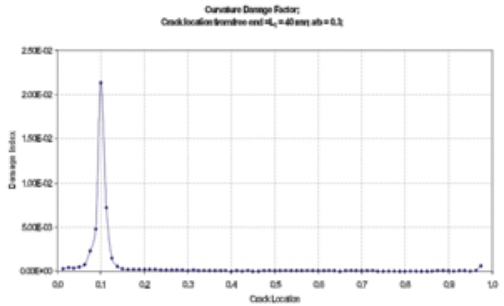

Fig. $7(c)$

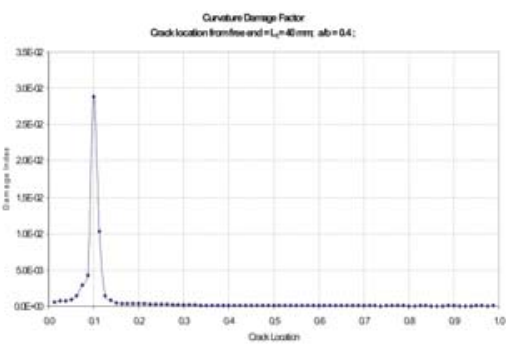

Fig. $7(d)$

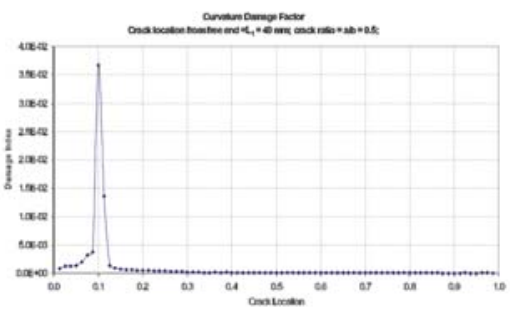

Fig. 7(e)
4. GAPPED SMOOTHING METHOD :

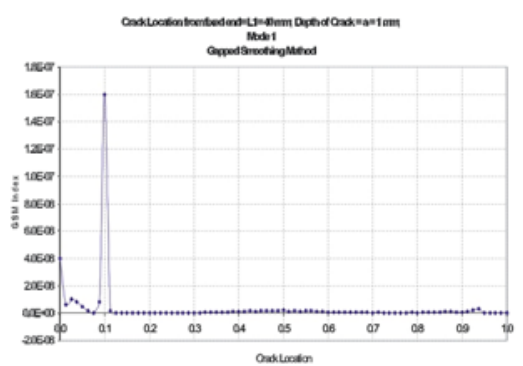

Fig. 8(a)

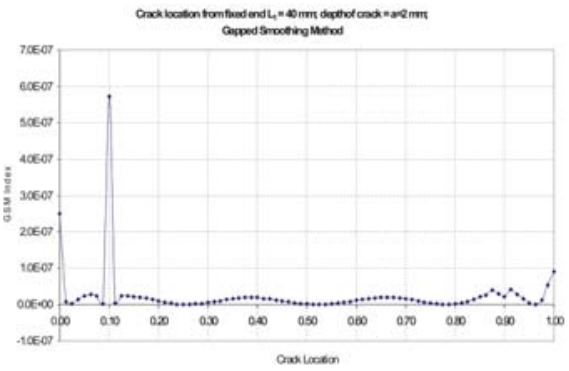

Fig. 8(b)

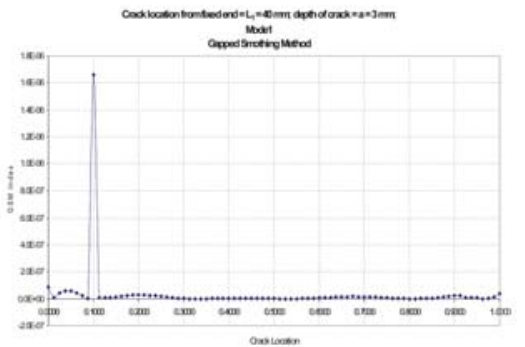

Fig. $8(\mathrm{c})$

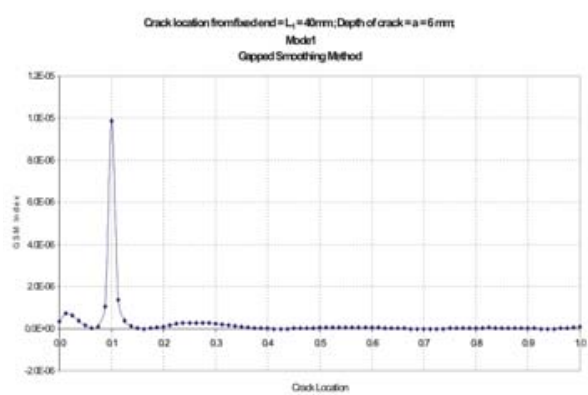

Fig. 8(d) 


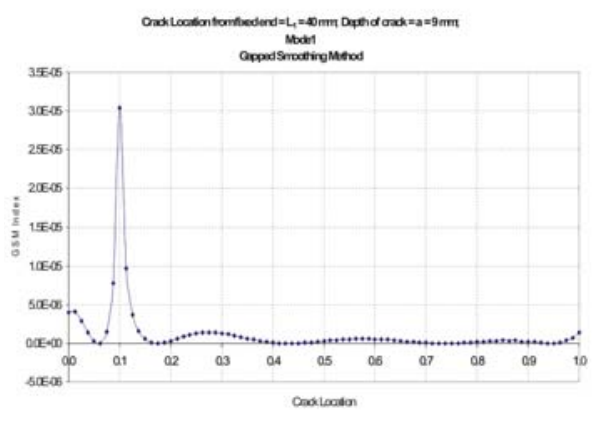

Fig. 8(e)

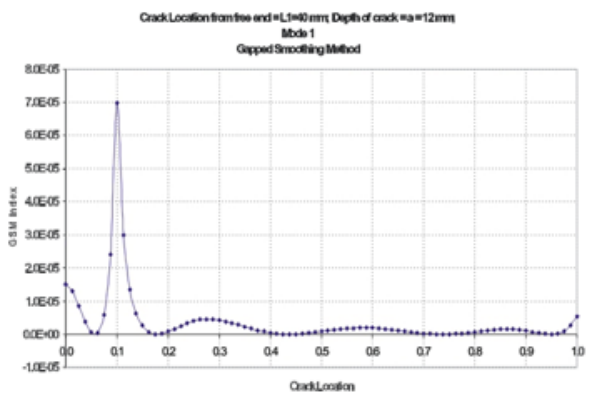

Fig. 8(f)

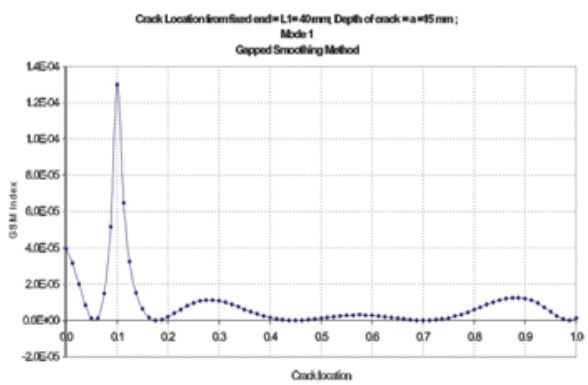

Fig. 8(g)

\section{THE STRAIN ENERGY METHOD :}

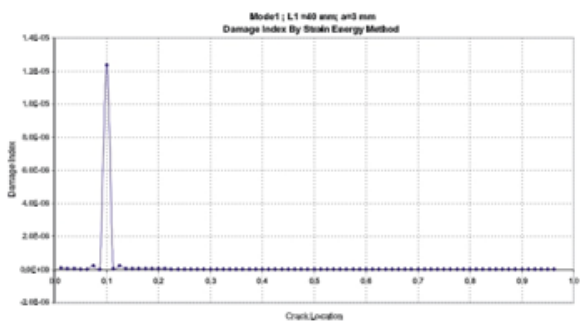

Fig. 9(a)

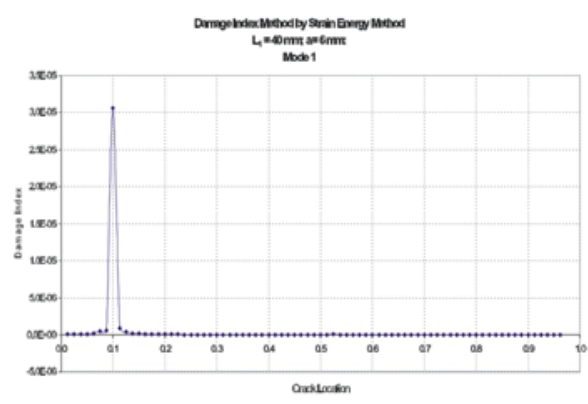

Fig. 9(b)

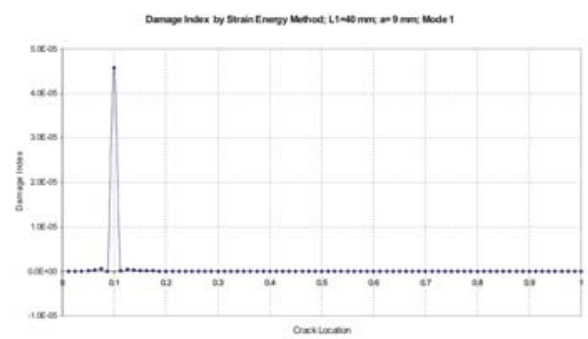

Fig. 9(c)

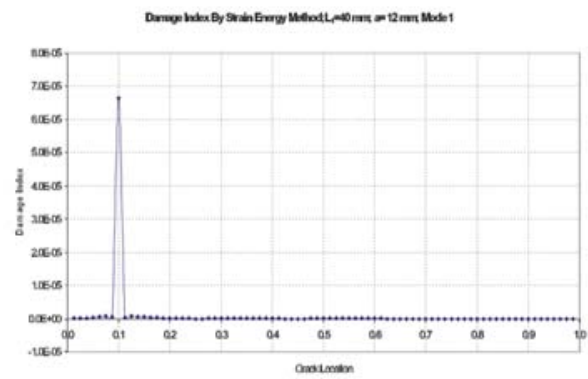

Fig. 9(d)

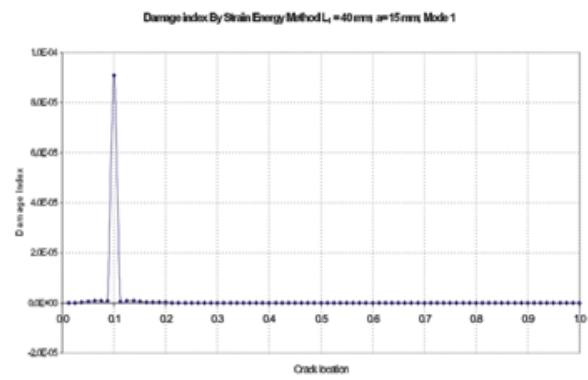

Fig. 9(e)

The effect of crack position on the sensitivity of natural frequencies and mode shapes of cantilever composite beam with a single edge crack has been studied. The following observations can be made from the above results.

1. For a given mode, the effect on the bending frequency and mode shape become more severe as the depth of the crack increases. 
2. If the position of crack is known information, one specific mode may be sufficient to obtain accurate results in the crack identification.

3. The add-on advantage by using the Gapped Smoothing Method and the Strain Energy method is that the data from the healthy beam are not required as the reference, thus simplifying the detection process.

4. Successful implementation of damage detection algorithms by using numerical procedure for crack identification of composite beam.

5. The dynamics-based damage detection approach using curvature mode shapes is useful method for structural health monitoring of composite structures and it is also an effective method of acquiring both the global and the local information of the structure.

The damage index values for the different damage algorithms are shown in Table 1.

Table 1. Damage index values

\begin{tabular}{|c|c|c|c|c|}
\hline $\begin{array}{c}\text { Crack } \\
\text { ratio }\end{array}$ & DIM & CDF & SEM & GSM \\
\hline 0.1 & 1.6913 & $8.87 \mathrm{e}-03$ & $1.23 \mathrm{e}-05$ & $1.66 \mathrm{e}-06$ \\
\hline 0.2 & 1.7201 & $1.62 \mathrm{e}-02$ & $3.06 \mathrm{e}-05$ & $9.87 \mathrm{e}-06$ \\
\hline 0.3 & 1.8133 & $2.13 \mathrm{e}-02$ & $4.57 \mathrm{e}-05$ & $3.04 \mathrm{e}-05$ \\
\hline 0.4 & 1.8671 & $2.88 \mathrm{e}-02$ & $6.64 \mathrm{e}-05$ & $6.99 \mathrm{e}-05$ \\
\hline 0.5 & 1.8969 & $3.67 \mathrm{e}-02$ & $9.07 \mathrm{e}-05$ & $1.30 \mathrm{e}-04$ \\
\hline
\end{tabular}

DIM : Damage Index Method

CDF : Curvature Damage Factor

SEM : Strain Energy Method

GSM : Gapped Smoothing Method

\section{CONCLUSION}

In summary, The dynamics-based approach has been widely used to identify damage. The frequencybased method indicates its usefulness in probing the presence of the defect, but it is relatively difficult to implement this method to locate/size the damage. Most of the above studies showed that the mode shapes-based method could be applied to detect damage type, size, and location in either large or small structures with some success. The change in frequencies of the few fundamental modes is more useful for identification of crack location. In real application, these changes in frequency values can be obtained by mounting sensors in the structure and the frequencies can be obtained by monitoring periodic measurements.
The approach based on dynamic response is relatively straight forwarded and easy to use, and it can be used as a viable technique for damage detection. Thus, the dynamics-based damage detection approach is a adopted in this study, and several damage detection algorithms are evaluated for composite beam-by using FEM software ANSYS.

In this study, a simple structure was considered, but this procedure can also be extended to identify and locate cracks in complex structures. The numerical data obtained from the above procedure is also useful for identification of crack depth approximately.

\section{REFERENCES}

[1] Adams, R.D., Cawley, P., Pye, C.J. and Stone, B.J. 1978. "A Vibration Technique for Nondestructively Assessing the Integrity of Structures," Journal of Mechanical Engineering Science, 20(2):93-100.

[2] Cawley, P. and Adams, R.D. 1979a. "The Localization of Defects in Structure from Measurements of Natural Frequencies," Journal of Strain Analysis, 2:49-57.

[3] Cawley, P. and Adams, R.D. 1979b. "A Vibration Technique for Nondestructive Testing of Fiber Composite Structures," Journal ofComposite Materials, 13(3):161-175.

[4] Gomez, A.J.M.M. and Silva, J.M.M. 1991. "On the use of Modal Analysis for Crack Identification," In: Proceeding of the 8th International Modal Analysis, FL, pp. 1108-1115.

[5] Salawu, O.S. 1997. "Detection of Structural Damage through Changes in Frequency: A Review,"Engineering Structures, 19:718-723.

[6] Messina, A., Williams, E.J. and Contursi, T. 1998. "Structural Damage Detection by a Sensitivity and Statistical-based Method," Journal of Sound and Vibration, 216(5):791-808.

[7] Zhou, Y., Tong, L. and Steven, G.P. 2000. "Vibration-based Model dependent Damage (Delamination) Identification and Health Monitoring for Composites Structures - A Review," Journal of Sound and Vibration, 230(2):357-378.

[8] Lee, Y.S. and Chung, M.J. (2000). A study on crack detection using eigen frequency test data. Computers and Structures, 77 (2000), 327-342. 
[9] Chinchalkar, S. (2001). Determination of crack location in beams using natural frequencies. Journal of Sound and Vibration 247(3), 417-429.

[10] Lestari, W. and Hanagud, S. 2001. "Detection of an Edge-notch Defect by using a Single Mode Based Methods,"In: Proceedings of 3rd International Workshop on SHM, pp. 1343-1355

[11] Kessler, S.S., Spearing, S.M., Atalla, M.J., Cesnik, C.E.S. and Soutis,C. 2002. "Damage Detection in Composite Materials using Frequency Response Methods," Composites Part B: Engineering,33(1):87-95.

[12] Lestari, W. and Qiao, P. 2005. "Damage Detection of Fiber-reinforced Polymer Honeycomb Sandwich Beams," J. Composite Structures,67(3):365-373

[13] Pandey, A.K., Biswas, M. and Samman, M.M. 1991. "Damage Detection from Changes in Curvature Mode Shapes," Journal ofSound and Vibration, 145(2):321-332.
[14] Stubb, N. and Kim, J.T. 1996. "Damage Localization in Structures without Baseline Modal Parameter," AIAA Journal,34: 1644-1649.

[15] Wahab, M.M.A. and De Roeck, G. (1999). Damage detection in bridges using modal curvatures: applications to a real damage scenario. Journal of Sound and Vibration, 226(2), 217-235.

[16[ Ratcliffe, C. P. and Bagaria, W. J. (1998). Vibration technique for locating delamination in a composite plates. AIAA Journal, 36, 1074-1077

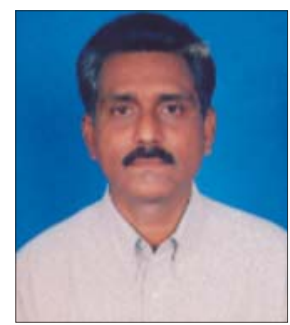

Mr. E. V. V. Ramanamurthy is a Research Scholar at JNTU, Hyderabad and an Assistant Professor at the Department of Mechanical Engineering, Sathyabama University, Chennai. A post-graduate from JNTU, Hyderabad, his specialization is Machine Design. He has over eighteen years of teaching experience. 